\title{
Theta coupling between V4 and prefrontal cortex predicts visual short-term memory performance
}

\author{
Stefanie Liebe ${ }^{1,5}$, Gregor M Hoerzer ${ }^{2}$, Nikos K Logothetis ${ }^{1,3}$ \& Gregor Rainer ${ }^{1,4}$ \\ Short-term memory requires communication between multiple brain regions that collectively mediate the encoding \\ and maintenance of sensory information. It has been suggested that oscillatory synchronization underlies intercortical \\ communication. Yet, whether and how distant cortical areas cooperate during visual memory remains elusive. We examined neural \\ interactions between visual area V4 and the lateral prefrontal cortex using simultaneous local field potential (LFP) recordings \\ and single-unit activity (SUA) in monkeys performing a visual short-term memory task. During the memory period, we observed \\ enhanced between-area phase synchronization in theta frequencies (3-9 Hz) of LFPs together with elevated phase locking of SUA \\ to theta oscillations across regions. In addition, we found that the strength of intercortical locking was predictive of the animals' \\ behavioral performance. This suggests that theta-band synchronization coordinates action potential communication between V4 \\ and prefrontal cortex that may contribute to the maintenance of visual short-term memories.
}

Every cognitive act entails the participation of multiple brain regions. In visual short-term memory, for example, visual information is initially encoded in sensory brain areas and then communicated to regions that mediate the retention, manipulation and retrieval of information. One prominent hypothesis that addresses the question of how communication between neural ensembles is achieved claims that neuronal oscillations support the timely coordination of neural activity between different brain regions ${ }^{1,2}$. Specifically, neuronal oscillations in the theta frequency band (3-9 Hz) have been suggested to underlie the interaction between neural ensembles during mnemonic processing 3 .

A line of evidence supporting this hypothesis stems from studies investigating the role of hippocampal theta in memory formation in rodents ${ }^{5-7}$. Additional evidence comes from studies measuring surface-based or intracortical electroencephalography (EEG) in human subjects. In these cases, memory performance correlates with an increase in theta power ${ }^{8-10}$. Moreover, enhanced theta synchrony between electric potentials recorded from memory-related areas has been observed ${ }^{11,12}$.

These findings raise the question of whether theta synchrony measured at the mesoscopic level of LFPs provides a basis for the timely coordination of spiking output between distant cortical areas that have been traditionally associated with the sensory encoding of visual information on the one hand and mnemonic processing on the other. Moreover, is the precision of coordination between these regions associated with changes in memory performance? To answer these questions, we studied neuronal interactions between the extrastriate visual area V4 and the lateral prefrontal cortex (IPF) while monkeys performed a visual memory task.

Although neural activity in V4 has been related to color and shape processing of visual objects ${ }^{13-16}$ and visual attention ${ }^{17}$, neural responses in IPF have been traditionally associated with working memory, that is, the short-term maintenance and manipulation of sensory information in memory tasks ${ }^{18,19}$. More recently, however, an increasing number of studies have found that the neural circuitry underlying short-term retention of sensory information likely entails earlier sensory cortical areas as well ${ }^{20}$. In these cases, both prefrontal regions ${ }^{21}$ and $\mathrm{V} 4$ have been linked to memory-related oscillatory synchrony. Specifically, theta oscillations are enhanced during the delay period of memory tasks in both IPF and V4 (ref. 22), and increased oscillatory theta synchrony is accompanied by a phase-dependent coding of visual stimuli retained in short-term memory ${ }^{23,24}$.

We observed enhanced phase locking between local field potentials recorded in V4 and IPF (inter-area LFP phase locking) that occurred in the theta range $(\sim 3-9 \mathrm{~Hz})$ during the memory period of a visual short-term memory task. Increased LFP-phase locking was associated with greater locking of spikes to the phase of the theta oscillations in the respective other area (inter-area spike-phase locking). Both inter-area LFP- and spike-phase locking were substantially higher for subsequent correctly remembered stimuli and were also predictive of session-to-session variations in task performance.

\section{RESULTS}

We simultaneously measured LFP and spiking activity in the dorsal portion of V4 and the dorsal and ventral lPF (Supplementary Fig. 1). During recordings, monkeys performed a delayed matching-tosample task (Fig. 1a) in which the monkeys had to retain information about a briefly presented visual stimulus $(250 \mathrm{~ms})$ over the course of a delay period $(1,500 \mathrm{~ms})$. The sample stimuli consisted of a set of natural images that were shown at different levels of image degradation (Supplementary Fig. 2). The stimulus conditions that were used

\footnotetext{
${ }^{1}$ Max Planck Institute for Biological Cybernetics, Department of Physiology of Cognitive Processes, Tuebingen, Germany. ${ }^{2}$ Institute for Theoretical Computer Science, Graz University of Technology, Graz, Austria. ${ }^{3}$ The University of Manchester, Faculty of Medical and Human Sciences, Manchester, UK. ${ }^{4}$ Department of Medicine, University of Fribourg, Switzerland. ${ }^{5}$ Present address: Department of Physiology, Pharmacology and Neuroscience, University College London, London, UK. Correspondence should be addressed to S.L. (s.liebe@ucl.ac.uk) or G.R. (gregor.rainer@unifr.ch).
} 
Figure 1 Experimental procedure, behavioral performance and power modulations in areas V4 and IPF during baseline and delay. (a) Events and their respective duration during the delayed matching-to-sample task. Monkeys had to fixate a small fixation spot $\left(2^{\circ}\right)$ on the screen; subsequently, a sample stimulus was shown for $250 \mathrm{~ms}$, which was followed by a delay period during which the monkeys had to retain fixation. Afterwards, a test stimulus was shown that either matched or did not match the sample stimulus (50\% match, 50\% non-match trials). (b) Psychophysical performance of both animals in the task using different levels of stimulus coherence for the sample stimulus. M1, monkey 1; M2, monkey 2. Error bars denote s.e.m. (c) Time-frequency maps of spectral power, normalized to baseline. PF, prefrontal cortex. (d) Changes in spectral power (measured as s.d.) during delay with respect to baseline.

have been described in detail in a previous study ${ }^{25}$. Both monkeys showed increased recognition performance as sample images became less degraded (Fig. 1b). In all subsequent analyses, the full noise condition was excluded unless otherwise stated.

First, we confirmed previous results on increased theta oscillations during visual memory. We compared the amplitude spectra of LFPs from a total of 131 sites recorded in V4 (monkey 1, 86; monkey 2, 45) and 117 sites in $\mathrm{PPF}$ (monkey 1, 74; monkey 2, 43) during the delay period of the task to those recorded during a baseline period before the sample stimulus onset. In both monkeys, the frequency spectra showed local increases in the theta band (3-9 Hz) in V4 and IPF during the delay period compared with during the baseline period (V4, $z>4.3, P<0.01$; IPF, $z>5.4, P<0.01$; Wilcoxon signed rank test for both monkeys; Fig. 1c,d and Supplementary Fig. 3).

\section{Enhanced inter-area theta phase synchrony during delay}

We subsequently investigated theta-band synchronization between V4 and IPF LFP sites during the delay period. We computed the phase locking value (PLV) ${ }^{26}$ between simultaneously recorded V4 and IPF channel pairs ( $N=332$ for monkey 1 and 175 for monkey 2$)$ for frequencies between 1 and $100 \mathrm{~Hz}$ (see Online Methods). A consistent phase relationship between V4-IPF LFP channel pairs during the delay period, but not during the baseline period, can be clearly seen in the oscillations of the single example pair (Fig. 2a) and was reflected in significantly enhanced PLV during the delay period in the theta range for many LFP pairs ( $P<0.01$; Fig. 2b,c and Supplementary Fig. 4).

We generally observed an increase in theta phase locking during the delay across all recorded LFP pairs (Fig. 2d and Supplementary Fig. 5). This was also consistent across recording sessions and locations (Supplementary Figs. 6 and 7) and could be replicated using a different spectral analysis (Supplementary Fig. 8). In general, the increase in phase locking was most prominent between 3 and $9 \mathrm{~Hz}$, which is consistent with both the frequency range in which we observed amplitude increases during the delay period and the frequency range that is traditionally associated with the theta band. Phase locking in theta started to increase toward the end of the sample stimulus presentation and was maintained throughout the entire delay period for $1.5 \mathrm{~s}$.

When assessing phase locking in individual LFP pairs, we found that 168 of 507 pairs showed significantly elevated phase locking during the delay $(P<0.001$ based on permutation tests; monkey 1,135 of 332 pairs; monkey 2, 33 of 175 pairs; $z$ test for significant proportion, $P<0.001)$. The proportion of pairs showing elevated phase locking was highest in theta $(3-9 \mathrm{~Hz}$ ), with $29 \%$ and $13 \%$ for monkeys 1 and 2 , respectively ( $z$ test for significant proportion, $P<0.01$ ). Although we generally observed that theta phase locking increased during the delay, we also observed significant decreases in theta locking in a small and nonsignificant proportion of pairs (monkey 1, 13 of 332 pairs; monkey 2, 12 of 175 pairs; $z$ test, $z<1.21, P>0.05$ ). a

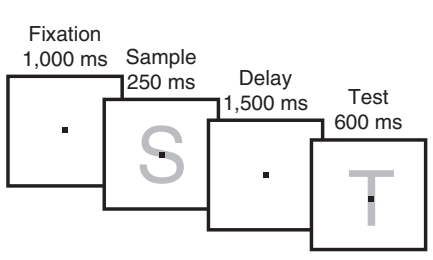

C

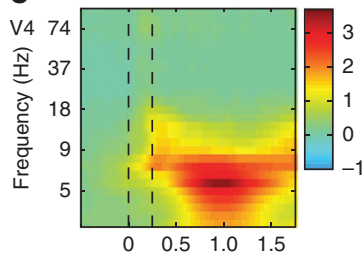

Time from sample onset (s)

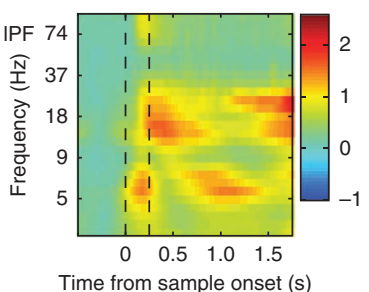

Time from sample onset (s) d b

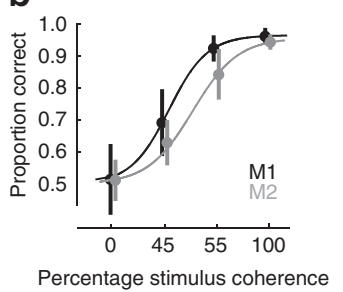

During the delay, no other frequency bands seemed to show systematic changes in phase locking. We found only a small proportion of pairs showing a significant increase in PLV during the delay in the beta $(16-36 \mathrm{~Hz})$ and gamma $(42-97 \mathrm{~Hz})$ frequency ranges. These proportions were significantly smaller than that for theta in both monkeys (monkey $1, P_{\text {theta }}=0.29, P_{\text {beta }}=0.07, P_{\text {gamma }}=0.12$; monkey 2, $P_{\text {theta }}=0.13, P_{\text {beta }}=0.04, P_{\text {gamma }}=0.06 ; \chi^{2}$ test, $\chi^{2}>12.3$, $P<0.001$ for all comparisons; Fig. 2 e).

We also examined the timing relationship of theta synchrony between V4 and IPF. We observed a negative phase difference between V4 and IPF across LFP pairs with a general time lag of approximately $-15 \mathrm{~ms}$ (Fig. 2f), which is consistent with the idea of prefrontal oscillations leading those in V4. Our nonzero phase lags imply direct interactions and asymmetric coupling between the regions rather than common input and bidirectional interactions ${ }^{2}$. The timeshifts are small enough to support direct interactions between V4 and lPF, especially considering the role of synchronous events in promoting fast and reliable postsynaptic depolarization ${ }^{27}$.

Next, we analyzed whether increases in theta-band phase locking between oscillatory LFP activity was associated with increases in spikephase locking. Each spike was assigned its respective analytic phase value of the simultaneous LFP oscillation during the baseline and delay period. We used Rayleigh's $z$ score as a test statistic to determine whether spiking of individual units was significantly locked to particular theta phases and generated histograms containing spiking probability as function of phase. Fits with von Mises density functions yielded estimates of the mean direction $\mu$ (that is, preferred phase) and the concentration $\kappa$ of spiking (that is, how peaked the spiking distribution is around the preferred phase). After normalizing for differences in spike rates between baseline and delay, we compared the proportion of significantly locked units, as well as median $\kappa$, between these task periods.

We first examined within-area spike-phase locking of V4 and prefrontal neurons during the baseline and delay periods (number of simultaneously recorded unit-channel pairs in V4, monkey 1 , 426; monkey 2, 175; lPF, monkey 1, 216; monkey 2, 181). Across all 
a

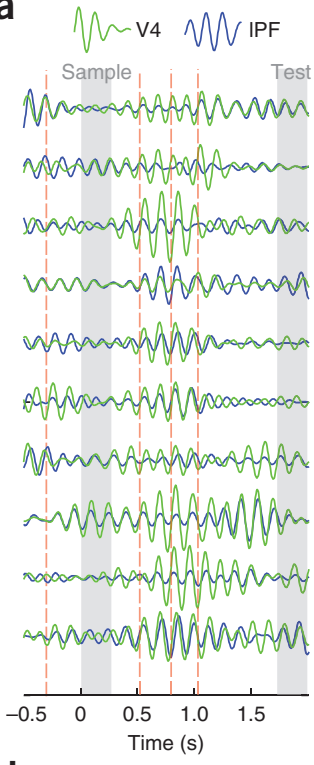

d

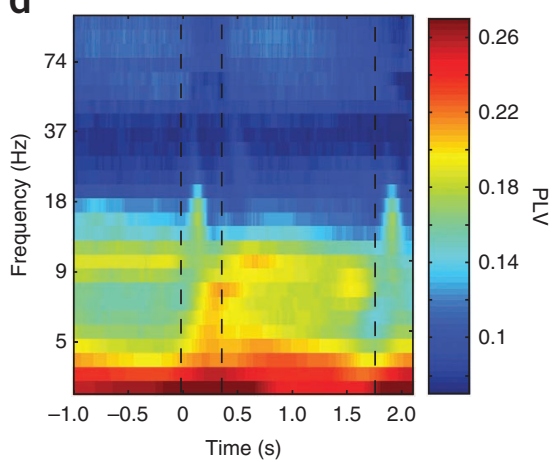

b
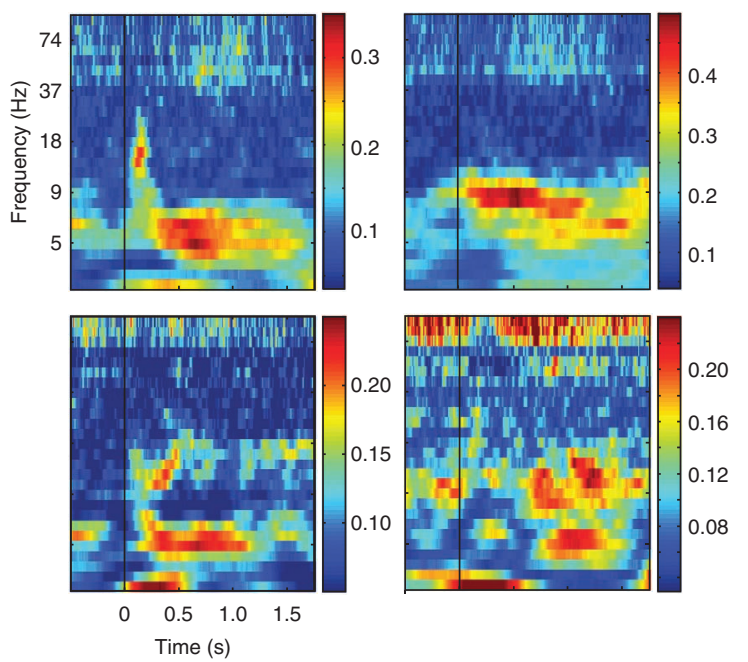

c
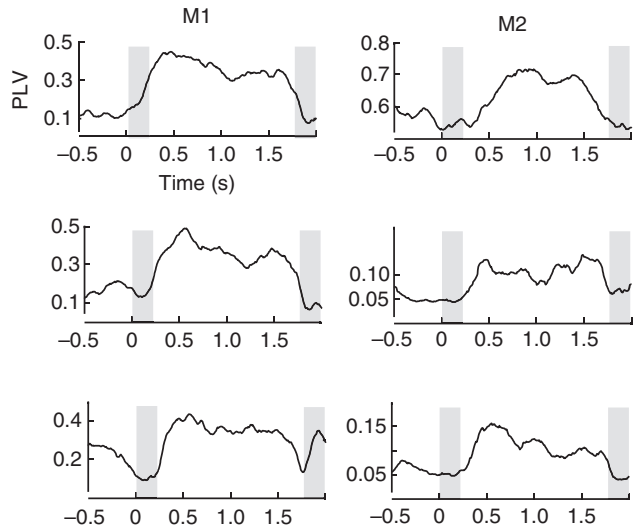

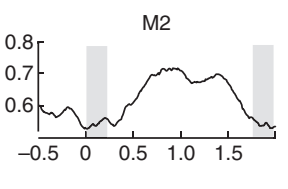

e

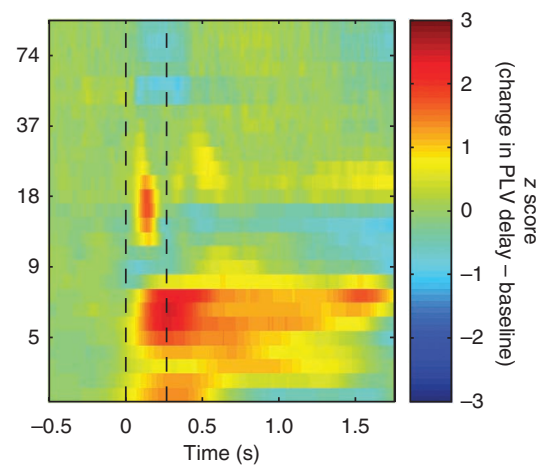

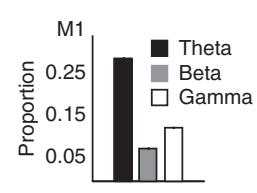

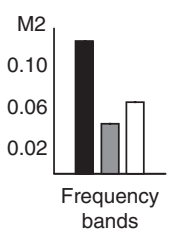

f
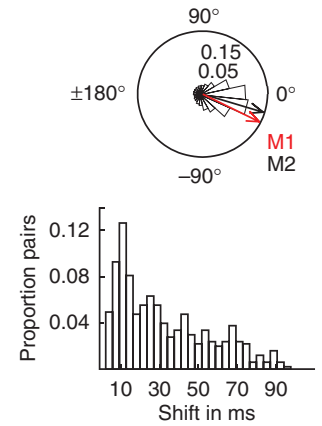

Figure 2 Phase locking between V4 and IPF in theta band. (a) Example of theta-filtered (at $6.8 \mathrm{~Hz}$ ) single-trial LFP traces from two simultaneously recorded V4 (green) and IPF (blue) channels illustrating consistent phase relationship during the delay period, but not during the baseline period (red dashed vertical lines are for illustration purposes only). Gray regions indicate the presentation intervals of the sample and test stimulus, respectively. (b) Representative examples of theta phase locking as measured by the PLV as a function of time and frequency for monkeys 1 (left) and monkey 2 (right). (c) Time-averaged PLV in theta as a function of time from additional examples. Note that the upper left trace corresponds to the example shown in a. (d) Time-frequency representation of the averaged raw PLV and normalized PLV difference ( $z$ score) between baseline and delay periods across all recorded pairs. Dashed vertical lines indicate the onset and offset of the sample stimulus. (e) Proportion of significantly locked pairs during the delay period as a function of frequency band for both monkeys. Significance thresholds were determined on the basis of a bootstrapping procedure comparing baseline and delay periods using $P<0.001$. (f) Distribution of phase differences and time shifts between all of the V4-PF pairs during the delay period in the frequency range of 3 to $9 \mathrm{~Hz}$. Arrows indicate the circular mean phase difference of monkeys 1 and 2.

recorded units, theta-band spike-phase locking was significantly higher during the delay period than during the baseline period (rank sum test comparing median $\kappa, z>2.7, P<0.01$ in both areas and monkeys). V4 units showed a greater enhancement in theta locking than prefrontal units (V4, 23\% increase; $1 \mathrm{PF}, 14 \%$ increase). Although this effect was observable across all theta frequencies $(3-9 \mathrm{~Hz})$ for one monkey ( $z>9.4, P<0.001$ for each region), the increase in spikephase locking was mainly present below $6 \mathrm{~Hz}$ in the other monkey ( $z>2.5, P<0.01$ for each region). Similar to the effect at the population level, we observed a significant increase in the proportion of significantly coupled units from baseline to delay in V4 (17\%) and IPF (30\%, $z>2.1, P<0.05$ for each region and monkey). These effects were evident in the median concentration parameters for all V4 and prefrontal units during the baseline and delay periods, as well as in the phase of firing probability histograms averaged across all significantly locked units in both regions $(P<0.01$; Fig. 3$)$.

Although these results are similar to previous studies ${ }^{23,24}$, the more interesting question is whether there is enhanced inter-regional spike-phase locking during the memory period of the task. If phase synchrony at the level of LFPs provides the timely basis for coordinating spiking output between both regions, our findings on increased LFP-phase locking between V4 and IPF during visual memory imply that higher spike-phase locking between regions could be the case.

To examine spike-phase locking between V4 and IPF, we analyzed the activity of 660 simultaneously recorded lPF LFP-V4 unit pairs (monkey 1, 458; monkey 2, 202) and 593 V4 LFP-lPF unit pairs (monkey 1, 335; monkey 2, 258). We found that the spike rate of single units in V4 and IPF was significantly modulated as a function of theta phase in the respective other area during the delay $(P<0.01$; Fig. 4a,b). We observed this effect across all V4 and prefrontal units (Wilcoxon signed rank test, baseline versus delay; V4 units, $z>3.5, P<0.01$; IPF, $z>3.4, P<0.01)$. Moreover, the increase in inter-area coupling was larger than the effect that we observed within regions. During delay, spike-phase locking between V4 units and prefrontal LFPs was enhanced by $31 \%$ and between prefrontal units and V4 LFPs by 19\% (Fig. 4c). Consistent with the effect at the population 
Figure 3 Enhanced intra-area spike-phase locking in theta during delay. $(\mathbf{a}, \mathbf{b})$ Phase of firing probability averaged across significantly locked V4 and prefrontal neurons $(P<0.01)$. The reference LFP cycle is shown below the histograms. Red and gray symbols mark the average preferred phase across the units and lines show the circular 95\% confidence intervals for V4 and prefrontal units, respectively. Plots depict two cycles for illustration purposes. (c,d) Median $\kappa$ values for baseline and delay for $\mathrm{V} 4$ and prefrontal units. Error bars denote \pm 34 th confidence intervals around median.

level, we found that the proportion of significantly locked V4 and prefrontal units was higher during the delay period than during the baseline period, with an average increase of $32 \%$ for V4 units and of $29 \%$ for $1 P F$ units ( $\chi^{2}$ test, $P<0.01$, both areas).

We next compared the strength and timing of phase locking between V4 units locked to IPF theta and IPF units locked to $\mathrm{V} 4$ theta. We examined in the average phase of firing probability histogram across all significantly locked V4 and IPF units along with the von Mises fits and the referenced oscillation cycle (V4, N=125; lPF, $N=86$; $P<0.01$; Fig. 4d). V4 units tended to fire toward the beginning of the theta peak, whereas prefrontal units had preferred phases toward the second half of the peak. This difference was well reflected in the distribution of preferred phases of V4 and IPF units, which were significantly different (mean preferred phase: $\mathrm{V} 4,72^{\circ}$; $1 \mathrm{PF}$, $108^{\circ} ; F=8.41, P<0.01$, Watson-Williams test; Fig. 4e). Finally, we compared the distribution of the concentration parameter $\kappa$ obtained from von Mises fits for each unit-LFP pair as well as the median $\kappa$ across significantly coupled pairs between V4 and lPF units (Fig. 4f). Notably, we obtained significantly higher concentration parameters for V4 units locked on IPF theta than for IPF units locked on V4 theta (monkey 1, $z=4.01$; monkey 2, $z=1.99 ; P<0.05$ ).

In summary, we found that V4 neurons exhibited significant phase locking to theta rhythms in IPF; similarly, IPF neurons exhibited phase
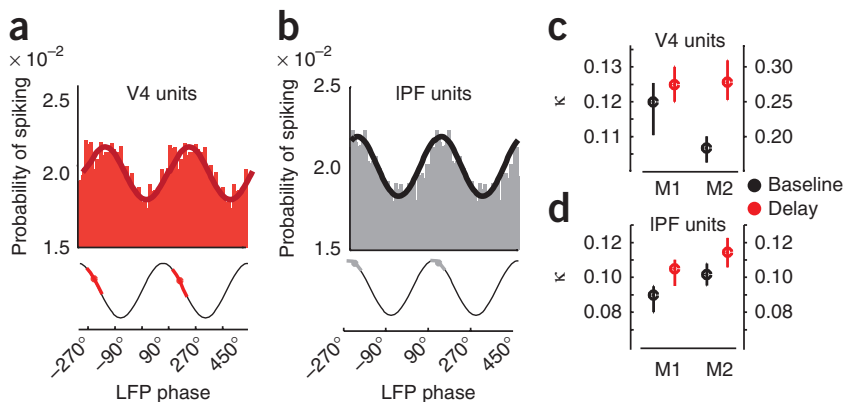

locking to V4 theta oscillations. Notably, the degree of phase locking was dependent on the task period: during the memory period, spikes in V4 and prefrontal cortex tended to occur more often around particular theta phases in the respective other area. The increase in spikephase locking during the delay period is consistent with enhanced intercortical locking at the level of LFPs between the regions. Thus, theta synchrony at the mesoscopic level of electric potentials may provide a mechanism by which spiking activity becomes more reliably coordinated in time across two distant cortical areas that are both involved in visual working memory.

\section{Performance-dependant inter-area theta coupling}

Thus far, our analyses revealed delay-specific increases in inter-cortical theta coupling both at the level of LFPs and SUA. Are changes in theta locking also associated with changes in memory performance? Given that our animals also performed the task with images that were degraded with visual noise, we were able to compare phase locking between correct and incorrect trials. All subsequent analyses use only trials during which sample stimuli were degraded (45\% stimulus coherence).

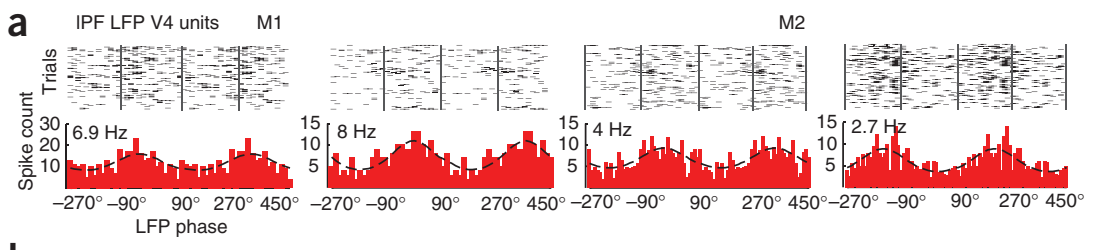

b

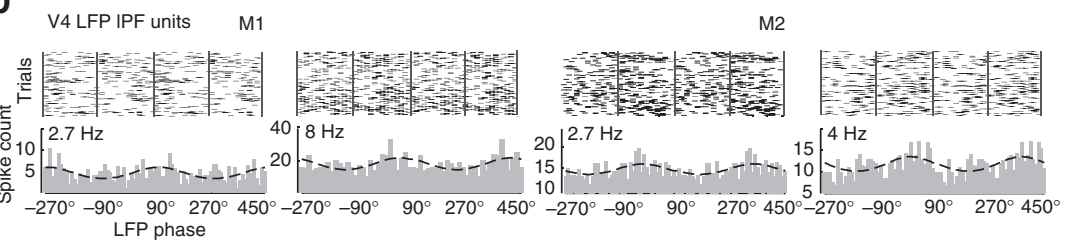

C

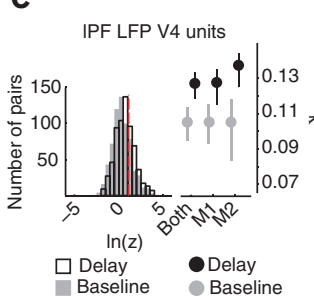

$\mathbf{d}_{\times 10^{-2}}$

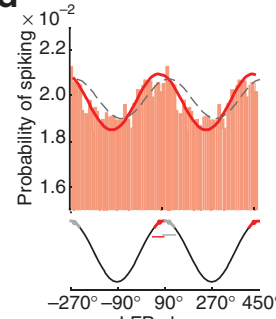

e
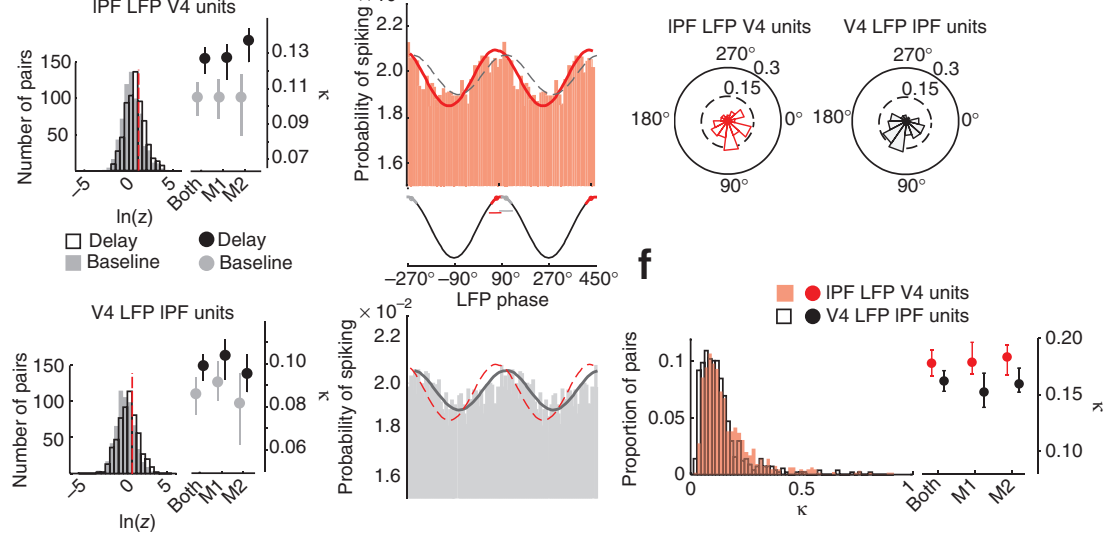

Figure 4 Inter-area theta phase locking of V4 and prefrontal neurons during visual memory. (a) Raster plots and summed spike histograms of V4 units significantly locked to IPF $(P<0.01)$, centered at the trough of the theta oscillation during delay period. Spike trains and histograms are plotted for two cycles for visual clarity. (b) Spiking activity of single units in IPF significantly locked to the theta rhythm in V4 $(P<0.01)$. (c) Histograms showing distribution of log-transformed Rayleigh's $z$ scores during baseline and delay. The red line indicates the significance at $\ln (z)>\ln (-\ln (p))$ for $P=0.05$. To the right of each histogram, median $\kappa$ values from von Mises fits of spike-phase distributions during baseline (gray) and delay (black) are shown. Error bars indicate $\pm 34 \%$ confidence intervals around the median. (d) Phase of firing probability and von Mises fits (V4, red, $\mu=1.26$ and $\kappa=0.08$; IPF, gray, $\mu=1.86$ and $\kappa=0.06$ ). In each histogram, the van Mises fit of the respective other area is also shown (dashed lines). Symbols mark the average preferred phase across the units and lines show the circular 95\% confidence intervals. (e) Circular distribution of preferred phases of V4 (red) and prefrontal (black) neurons. (f) Distribution of concentration parameter $\kappa$ for all pairs of simultaneously recorded V4 units and prefrontal LFPs as well as prefrontal units and V4 LFPs and median $\kappa$ values for V4 units locked to IPF theta and vice versa (for pairs statistically significant at $P<0.05$ ). Error bars denote \pm 34 th confidence intervals around median. 
Figure 5 Theta-phase locking during the delay is higher for correct than for incorrect trials. (a) Phase locking (average across all pairs) per frequency during the course of the task.

(b) Normalized (Norm.) difference (d') of correct minus incorrect trials during the delay period. (c) Normalized difference $\left(d^{\prime}\right)$ in phase locking between correct and incorrect trials averaged across the delay per frequency for pairs from monkey 1 (left) and monkey 2 (middle). Error bands correspond to \pm s.d. (d) Level of significance $(1-\log (P))$ for comparison between $d^{\prime}$ from original and shuffled datasets per frequency. (e) Median $\kappa$ estimates from phaseof-firing probabilities of correct and incorrect trials for V4 neurons (top) and prefrontal neurons (bottom, locking was calculated for significantly locked pairs, $P<0.01$ ). Error bars correspond to $\pm 34^{\text {th }}$ percentile around the median. (f) Phase of firing probability histogram of V4 neurons and prefrontal neurons for correct trials aligned to preferred phase per neuron and then averaged across neurons. Lines correspond to von Mises fits to correct (straight) and incorrect (dashed) spike histograms. (g) Distribution of preferred phase of V4 neurons and prefrontal neurons in correct and incorrect trials.

We first computed phase locking between LFP pairs and spike-LFP pairs separately for correct and incorrect trials. Specifically, we computed the normalized difference in phase locking $\left(d^{\prime}\right)$ between groups for which we randomly assigned correct or incorrect trials (shuffled $\mathrm{d}^{\prime}$ ) and compared them to the original dataset (original $\mathrm{d}^{\prime}$ ). We observed that theta phase locking was significantly elevated during the delay phase of trials in which the monkeys correctly identified the previously shown stimulus compared with trials in which the monkeys failed to do so $(P<0.01$ for 3-9 Hz, in each monkey). We also observed larger differences in phase locking during the early and late phase of the delay (Fig. 5a-d).

Several studies have found that prefrontal neurons can exhibit either early- or late-delay activity, and it has been suggested that they represent different memory processes, with early-delay activity corresponding to sensory maintenance of previously presented stimuli and late-delay activity representing the maintenance of the expected upcoming stimulus ${ }^{28}$. Similarly, early-delay versus late-delay theta synchronization might represent different memory processes that are both involved in successful memory performance.

The increase in LFP phase locking was also associated with an increase in spike-phase locking for correct compared with incorrect trials. This effect seemed to be greater for V4 than for prefrontal cortex units (Fig. 5e).
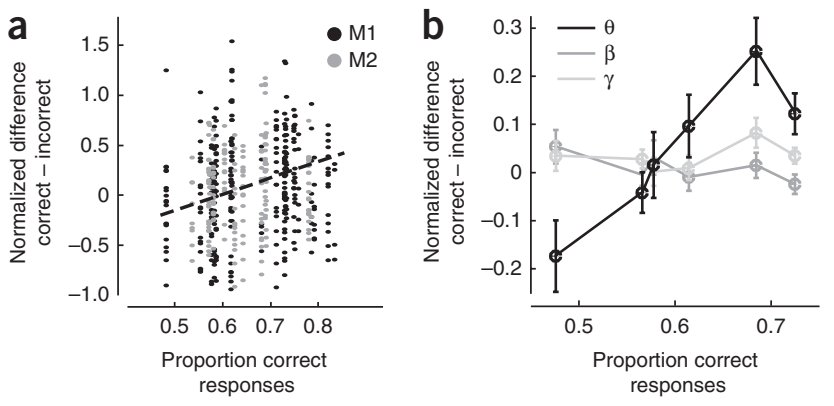
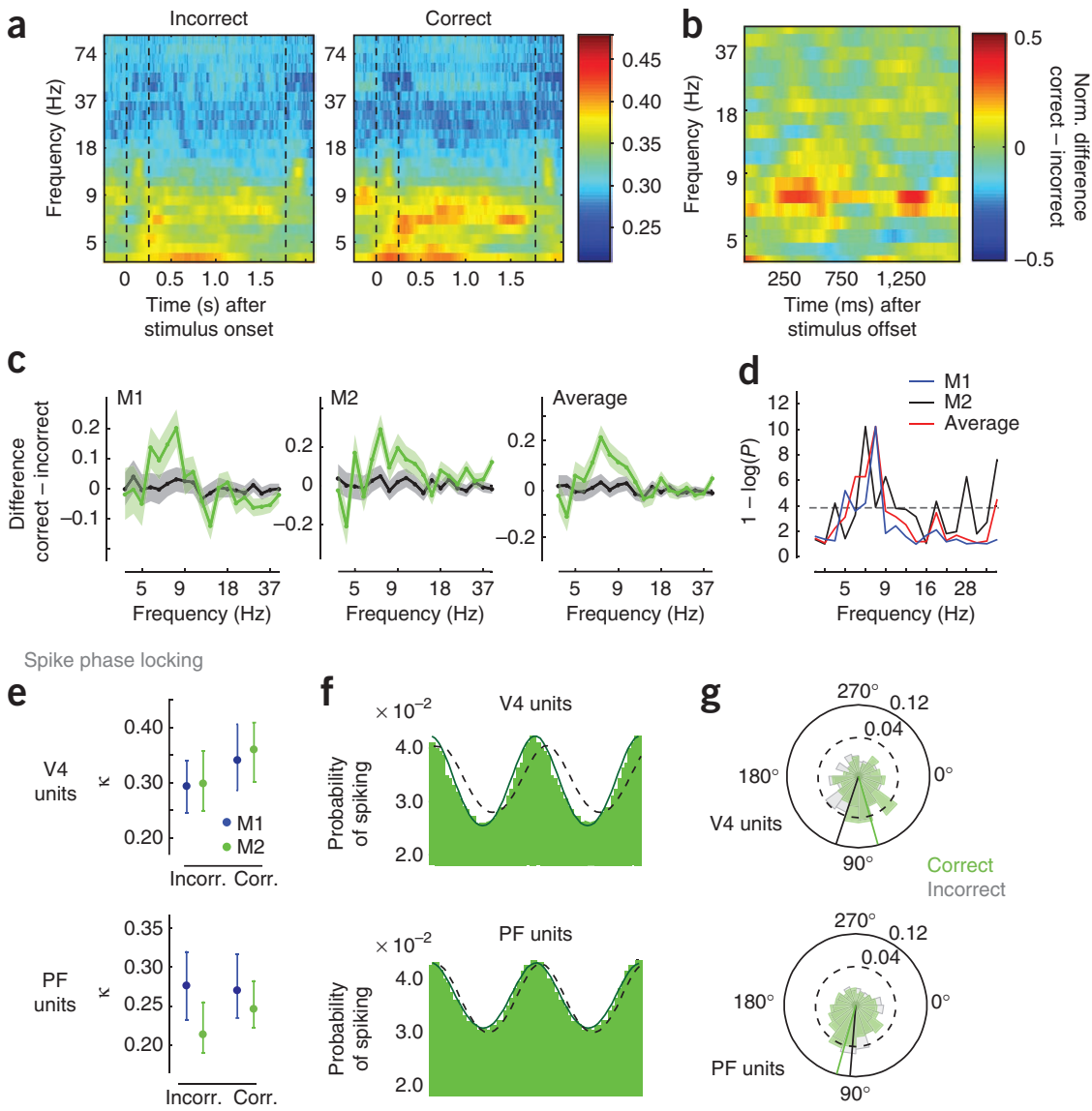

Although median $\kappa$ values were significantly higher for correct compared with incorrect trials in V4 in both monkeys (signed rank test, $z>2.95, P<0.001$ in each monkey), this was only true in one monkey in $\mathrm{lPF}$ (signed rank test: monkey $1, z=0.37, P>0.05$; monkey $2, z=2.1$, $P<0.05)$. In addition, we paired $\mathrm{d}^{\prime}$ values of $\mathrm{V} 4$ neurons recorded at V4 site $\mathrm{X}$ that were locked on prefrontal LFP site $\mathrm{Y}$ with $\mathrm{d}^{\prime}$ values of prefrontal neurons recorded at site $\mathrm{Y}$ that were locked at $\mathrm{V} 4$ site $\mathrm{X}$. We found that V4 neurons exhibited higher $\mathrm{d}^{\prime}$ values than prefrontal neurons recorded at the respective other LFP site in a significant majority of pairs (binomial test, $P<0.02$, for each monkey).

In addition to an increase in spike-phase locking during correct trials, we also observed differences in the timing relationship of spiking and theta oscillations between correct and incorrect trial types (Fig. 5f,g). Preferred phases were significantly different in correct versus incorrect trials for $\mathrm{V} 4$ neurons (mean phase: correct, $71.5^{\circ}$; incorrect, $\left.103.5^{\circ} ; F=16.69, P<0.001\right)$, but not for prefrontal cortex neurons (mean phase: correct, $105.1^{\circ}$; incorrect, $94.4^{\circ} ; F=2.1, P>0.05$ ).

In our previous analyses, we found that, on average, correct identification was associated with increases in theta-band phase locking relative to incorrect trials.

Figure 6 Correlation of phase locking with performance across single sessions. (a) Scatter plot showing normalized difference between correct and incorrect responses ( $y$ axis) as a function of the proportion of correct responses per session. For every session, the performance measure was paired with $d^{\prime}$ values of all pairs recorded during that session. Black symbols correspond to monkey 1 and gray symbols to monkey 2. (b) $d^{\prime}$ values binned into equally spaced percentiles of proportion of correct responses for three frequency bands (theta, black; beta, dark gray; gamma, light gray). Error bars correspond to \pm s.e.m. 
Does this difference co-vary with changes in performance across single sessions? We addressed this question by correlating $\mathrm{d}^{\prime}$ values per pair with the proportion of correct responses per session (Fig. 6). Indeed, $\mathrm{d}^{\prime}$ values and performance were positively correlated; that is, larger (positive) $\mathrm{d}^{\prime}$ values occurred during sessions with higher performance. This effect was again most dominant in the theta frequency range (rank correlations: monkey 1 and monkey $2, \rho_{\text {theta }}=0.18$ and $0.14, P<0.05 ; \rho_{\text {beta }}=0.11$ and $-0.06, P>0.05 ; \rho_{\text {gamma }}=0.01$ and $0.11, P>0.05)$.

\section{DISCUSSION}

\section{Delay-specific theta synchrony between V4 and IPF}

We found that LFP signals from V4 and prefrontal cortex exhibit a consistent phase relationship during the retention period that was substantially reduced during visual fixation and that occurred predominantly in the theta frequency range. This suggests that neural interaction based on oscillatory theta synchrony between two distant cortical regions is related to the maintenance and possible communication of visual information during visual short-term memory. Our findings are consistent with those of previous studies recording human EEG or intracortical LFP that showed increased theta power in either frontal or occipital regions during memory processing $9,23,29$, as well as previous observations of increased theta coherence between EEG signals from frontal and occipital regions in human subjects ${ }^{12,30}$. Taken together, our findings support the notion that prefrontal and visual cortical areas are part of a large-scale network that is involved in visual memory processing in primates ${ }^{20,31}$.

For many V4-lPF pairs, the synchronous theta oscillations in V4 and $\mathrm{PPF}$ were shifted in time by about $10-15 \mathrm{~ms}$, which is about an order of magnitude shorter than the cycle length of the underlying theta oscillations (120-250 ms). It has been suggested that phase shifts occur in this time range to account for integration times between different areas, such that presynaptic firing affects postsynaptic neurons at their peak depolarization phase, that is, during their most excitable state $2,32,33$. This could also be the case for our findings, where, for example, V4 spiking was structured to affect downstream areas, including the lPF, during their most excitable period or vice versa.

Similar to changes in neural synchrony at the level of LFPs, we found that inter-regional spike-phase locking was also enhanced during retention. At first, this finding might not seem surprising given that spikes in each area were phase-locked to their own theta rhythm. Thus, if theta oscillations between the areas become synchronized during the delay, spike-phase locking across areas is likely to occur. Nonetheless, the important thing to keep in mind is that we found higher interregional LFP phase locking, without which inter-regional spike-phase locking is not possible. This suggests that the increase in LFP-based theta synchrony mediates increased spike-phase locking between both regions. The importance of inter-regional spike-phase locking is further supported by the fact that memory-related enhancement in theta spike-phase locking was stronger between than within areas.

Spiking activity in V4 was more strongly locked to prefrontal theta than vice versa. This could imply that prefrontal regions are more involved in generating and sustaining theta oscillations during memory processing, as V4 spiking appears to be more sensitive to prefrontal theta than prefrontal spiking to V4 theta. However, if phase alignment of presynaptic spikes to postsynaptic membrane potential oscillations is the mechanism that establishes synchronous input into an area and therefore increases the probability of postsynaptic firing $2,33,34$, our observations could also indicate that V4 spiking may be consequently more effective in driving prefrontal activity. Nevertheless, the asymmetry in spike-phase locking between the regions implies a possible directedness in the interaction and communication pattern between the regions, of which the exact nature and timing relationship remain to be established.

\section{Theta coupling correlates well with memory performance}

Another important observation was that theta synchrony of LFPs between V4 and IPF predicted subsequent recognition of visual stimuli in each session and correlated well with session-to-session variations in memory performance. These findings are consistent with observations relating the strength of theta coupling to memory formation in humans using magneto- or electroencephalography ${ }^{35-37}$. Notably, we found that successful recognition was also associated with tighter coordination of spike timing with theta oscillations in the respective other region. Thus, our findings not only confirm a direct functional link between spike timing relative to theta oscillation and memory performance, but also extend for the first time, at least to the best of our knowledge, this principle to long-range interactions between distant neocortical areas. In addition, our results are consistent with the hypothesis that phase alignment between rhythmically active neural ensembles promotes effective communication ${ }^{2,4}$. Thus, theta phase synchronization may enhance memory performance by facilitating communication between V4 and lPF.

Both V4 and prefrontal units had their preferred phase shortly before and after the peak of the theta oscillations, respectively, that is, near the depolarized phase of the oscillation. Although spiking in a depolarized state is generally more likely to occur, these conditions have been shown to be ideal for inducing long-term potentiation in hippocampal structures ${ }^{38}$. Studies of the relationship between theta oscillation and synaptic plasticity in hippocampus suggest that only information of the appropriate phase is stored/encoded into memory ${ }^{7,39,40}$. Thus, long-term potentiation is thought to promote the encoding of sensory information into memory (also see ref. 4). Although our results do not provide direct evidence for this hypothesis, theta band synchronization between V4 and lPF might coordinate spiking output in a way that is ideal for the stimulus to undergo memory encoding.

\section{Is theta synchrony related to memory or decision processes?}

Given that our task involves the monkeys' decision of whether a previously presented sample stimulus matched a test stimulus shown after a delay, we must ask whether theta synchrony could be related to this decision. Several factors argue against this hypothesis. First, theta synchrony does not seem to increase as the test stimulus (or the decision) is approaching, which is often observed in preparatory and decision-related activity ${ }^{41}$. Second, the timing of the delay was slightly variable (by around $\pm 25 \mathrm{~ms}$ ), such that the monkeys could not precisely 'measure' or prepare themselves for when they would form the decision. Third, we observed increased theta phase locking with decreasing image degradation (Supplementary Fig. 9), although the monkeys were similarly preparing for the (lever release) decision, as only correct trials were analyzed in this comparison. The dependency on stimulus degradation suggests that theta phase locking between V4 and IPF during the delay is also modulated by visual information content.

The last analysis points to a potentially important role of visual identification in generating theta coupling. As noise increases, identification is less reliable, which could lead to lower theta coupling. The subsequent lack of memory processing could therefore only have a secondary role. Disentangling the respective contribution of either of these two processes for generating theta coupling will need to be investigated in the future. 
Finally, our findings are also compatible with computational models proposing phase-dependent coding as a means of storing and retrieving information in and from memory ${ }^{42}$, as well as with more recent models on synaptic mechanisms that underlie working memory ${ }^{43,44}$. According to these models, visual information is not exclusively stored by selective and sustained spiking activity during the delay period. Instead, information is thought to be maintained by calcium-mediated short-term synaptic facilitation and reactivated by population spikes, that is, synchronously spiking neural ensembles, at a rate corresponding to theta oscillations ${ }^{43}$. Along these lines, oscillatory synchrony has been recently linked to the ability to flexibly route information between neural populations ${ }^{45}$.

Taken together, our findings suggest that theta synchrony mediates the timed cooperation between neural ensembles in distant cortical areas. Increased coupling during successful retention may therefore reflect more efficient and selective routing and gating of information during short-term memory and might also promote encoding of information into memory. Ultimately, our observations are consistent with the proposition that oscillatory synchrony, and particularly phase synchronization, is a general principle of integrating information in and between different cortical and subcortical areas during complex cognitive processing.

\section{METHODS}

Methods and any associated references are available in the online version of the paper at http://www.nature.com/natureneuroscience/.

Note: Supplementary information is available on the Nature Neuroscience website.

\section{ACKNOWLEDGMENTS}

We would like to thank J. Macke for discussions regarding analyses, and T. Mrsic-Flogel and J. Macke for useful comments on the manuscript. We also thank A.-L. Keller for help with histology. S.L. was supported by the Deutsche Forschungsgemeinschaft Sonderforschungsbereich 550 and a doctoral Fellowship of the Max Planck Society. G.M.H. was supported by projects FP7-231267 ("SelfOrganized Recurrent Neural Learning for Language Processing”, ORGANIC) and FP7-506778 (Pattern Analysis, Statistical Modeling and Computational Learning, PASCAL2 ) of the European Union. G.R. is a European Science Foundation European Young Investigator.

\section{AUTHOR CONTRIBUTIONS}

S.L. and G.R. designed the experiments. S.L. conducted the experiments. S.L. and G.M.H. analyzed the data. S.L. wrote the manuscript with contributions from G.M.H. and G.R. N.K.L. and G.R. supervised the study.

\section{COMPETING FINANCIAL INTERESTS}

The authors declare no competing financial interests.

Published online at http://www.nature.com/natureneuroscience/.

Reprints and permissions information is available online at http://www.nature.com/ reprints/index.html

1. Buzsáki, G. \& Draguhn, A. Neuronal oscillations in cortical networks. Science 304, 1926-1929 (2004).

2. Fries, P. A mechanism for cognitive dynamics: neuronal communication through neuronal coherence. Trends Cogn. Sci. 9, 474-480 (2005).

3. Buzsáki, G. The hippocampo-neocortical dialogue. Cereb. Cortex 6, 81-92 (1996).

4. Fell, J. \& Axmacher, N. The role of phase synchronization in memory processes. Nat. Rev. Neurosci. 12, 105-118 (2011).

5. Jones, M.W. \& Wilson, M.A. Theta rhythms coordinate hippocampal-prefrontal interactions in a spatial memory task. PLoS Biol. 3, e402 (2005).

6. O'Keefe, J. Hippocampus, theta, and spatial memory. Curr. Opin. Neurobiol. 3, 917-924 (1993)

7. Buzsáki, G. Theta oscillations in the hippocampus. Neuron 33, 325-340 (2002).

8. Kahana, M.J. et al. Human theta oscillations exhibit task dependence during virtual maze navigation. Nature 399, 781-784 (1999).

9. Raghavachari, S. et al. Gating of human theta oscillations by a working memory task. J. Neurosci. 21, 3175-3183 (2001).
10. Tesche, C.D. \& Karhu, J. Theta oscillations index human hippocampal activation during a working memory task. Proc. Natl. Acad. Sci. USA 97, 919-924 (2000).

11. Rutishauser, U. et al. Human memory strength is predicted by theta-frequency phase-locking of single neurons. Nature 464, 903-907 (2010).

12. Sarnthein, J. et al. Synchronization between prefrontal and posterior association cortex during human working memory. Proc. Natl. Acad. Sci. USA 95, 7092-7096 (1998).

13. Orban, G.A. Higher order visual processing in macaque extrastriate cortex Physiol. Rev. 88, 59-89 (2008).

14. Pasupathy, A. Neural basis of shape representation in the primate brain. Prog. Brain Res. 154, 293-313 (2006).

15. Zeki, S. The representation of colours in the cerebral cortex. Nature $\mathbf{2 8 4}, \mathbf{4 1 2 - 4 1 8}$ (1980).

16. Liebe, S., Logothetis, N.K. \& Rainer, G. Dissociable effects of natural image structure and color on LFP and spiking activity in the lateral prefrontal cortex and extrastriate visual area V4. J. Neurosci. 31, 10215-10227 (2011).

17. Fries, P. et al. Modulation of oscillatory neuronal synchronization by selective visual attention. Science 291, 1560-1563 (2001).

18. Miller, E.K. \& Cohen, J.D. An integrative theory of prefrontal cortex function. Annu. Rev. Neurosci. 24, 167-202 (2001).

19. Fuster, J.M. \& Alexander, G. Neuron activity related to short-term memory. Science 173, 652 (1971)

20. Pasternak, T. \& Greenlee, M.W. Working memory in primate sensory systems. Nat. Rev. Neurosci. 6, 97-107 (2005).

21. Pesaran, B. et al. Temporal structure in neuronal activity during working memory in macaque parietal cortex. Nat. Neurosci. 5, 805-811 (2002).

22. Hoerzer, G.M. et al. Directed coupling in local field potentials of macaque v4 during visual short-term memory revealed by multivariate autoregressive models. Front. Comput. Neurosci. 4, 14 (2010).

23. Lee, $\mathrm{H}$. et al. Phase locking of single neuron activity to theta oscillations during working memory in monkey extrastriate visual cortex. Neuron 45, 147-156 (2005)

24. Siegel, M., Warden, M.R. \& Miller, E.K. Phase-dependent neuronal coding of objects in short-term memory. Proc. Natl. Acad. Sci. USA 106, 21341-21346 (2009).

25. Liebe, S. et al. Color and shape interactions in the recognition of natural scenes by human and monkey observers. J. Vis. 9, 14 (2009).

26. Lachaux, J.P. et al. Measuring phase synchrony in brain signals. Hum. Brain Mapp. 8, 194-208 (1999)

27. Azouz, R. \& Gray, C.M. Adaptive coincidence detection and dynamic gain control in visual cortical neurons in vivo. Neuron 37, 513-523 (2003).

28. Rainer, G. \& Miller, E.K. Timecourse of object-related neural activity in the primate prefrontal cortex during a short-term memory task. Eur. J. Neurosci. 15, 1244-1254 (2002).

29. Schack, B. et al. Phase-coupling of theta-gamma EEG rhythms during short-term memory processing. Int. J. Psychophysiol. 44, 143-163 (2002).

30. Weiss, S., Muller, H.M. \& Rappelsberger, P. Theta synchronization predicts efficient memory encoding of concrete and abstract nouns. Neuroreport 11, 2357-2361 (2000).

31. Klimesch, W. Memory processes, brain oscillations and EEG synchronization Int. J. Psychophysiol. 24, 61-100 (1996).

32. Tiesinga, P., Fellous, J.M. \& Sejnowski, T.J. Regulation of spike timing in visual cortical circuits. Nat. Rev. Neurosci. 9, 97-107 (2008).

33. Volgushev, M., Chistiakova, M. \& Singer, W. Modification of discharge patterns of neocortical neurons by induced oscillations of the membrane potential. Neuroscience 83, 15-25 (1998).

34. Womelsdorf, T. et al. Modulation of neuronal interactions through neuronal synchronization. Science 316, 1609-1612 (2007).

35. Fuentemilla, L. et al. Theta-coupled periodic replay in working memory. Curr. Biol. 20, 606-612 (2010)

36. Fell, J. et al. Phase-locking within human mediotemporal lobe predicts memory formation. Neuroimage 43, 410-419 (2008).

37. Guderian, S. \& Duzel, E. Induced theta oscillations mediate large-scale synchrony with mediotemporal areas during recollection in humans. Hippocampus 15, 901-912 (2005).

38. Pavlides, C. et al. Long-term potentiation in the dentate gyrus is induced preferentially on the positive phase of theta-rhythm. Brain Res. 439, 383-387 (1988).

39. Huerta, P.T. \& Lisman, J.E. Bidirectional synaptic plasticity induced by a single burst during cholinergic theta oscillation in Cal in vitro. Neuron 15, 1053-1063 (1995).

40. Lynch, M.A. Long-term potentiation and memory. Physiol. Rev. 84, 87-136 (2004).

41. Shadlen, M.N. \& Newsome, W.T. Neural basis of a perceptual decision i the parietal cortex (area LIP) of the rhesus monkey. J. Neurophysiol. 86, 1916-1936 (2001).

42. Jensen, O. \& Lisman, J.E. Hippocampal sequence-encoding driven by a cortical multi-item working memory buffer. Trends Neurosci. 28, 67-72 (2005).

43. Mongillo, G., Barak, O. \& Tsodyks, M. Synaptic theory of working memory. Science 319, 1543-1546 (2008).

44. Mehta, M.R., Quirk, M.C. \& Wilson, M.A. Experience-dependent asymmetric shape of hippocampal receptive fields. Neuron 25, 707-715 (2000).

45. Akam, T. \& Kullmann, D.M. Oscillations and filtering networks support flexible routing of information. Neuron 67, 308-320 (2010). 


\section{ONLINE METHODS}

Animals and recordings. Two adult male monkeys (Macaca mulatta) participated in the experiments. All studies were approved by the local authorities (Regierungspräsidium, Tübingen Referat 35, Veterinärwesen Leiter Dr. Hilmers) and were in full compliance with applicable guidelines (EUVD 86/609/EEC) for the care and use of laboratory animals. SUA and the LFPs were recorded from two recording chambers placed on the surface of the skull based on stereotaxic coordinates. The Horsley-Clarke coordinates for the centers of the recording chambers for monkey 1 were -6.5 anterior-posterior, -29.7 mediolateral (V4); and -33 anterior-posterior, -23.7 medio-lateral (prefrontal cortex). For monkey 2, the coordinates for the chambers were -5.2 anterior-posterior, -29.9 medio-lateral (V4); and -34.5 anterior-posterior, -22.6 medio-lateral (prefrontal cortex). The implantation and surgical procedures that we used were described previously ${ }^{23}$.

Neural signals were measured using two custom-made micro drives mounted on a plastic grid (Crist Instruments). In each recording session, 4-6 tungsten microelectrodes (UEWLGDSMNN1E, FHC) were manually lowered in pairs with a minimal separation of $0.5 \mathrm{~mm}$ between the electrodes. The impedance of the microelectrodes was approximately $1 \mathrm{M} \Omega$ at $1 \mathrm{kHz}$. The signal from each electrode was preamplified (factor 20, Thomas Recording) and signals from each area were referenced against their recording chamber. The analog signal was split into two signals and filtered and amplified separately (BAK Electronics) to extract SUA and the LFP responses. The LFP was obtained by band-pass filtering the signal between $0.1 \mathrm{~Hz}$ and $300 \mathrm{~Hz}$ and digitizing with a sampling rate of $4,464 \mathrm{~Hz}$.

The spiking activity was obtained by band-pass filtering the signal between $300 \mathrm{~Hz}$ and $4 \mathrm{kHz}$ and digitizing with a sampling rate of $22.231 \mathrm{kHz}$. Single units were extracted from the spiking activity using standard spike sorting routines (Offline Sorter, Plexon). To ensure stable recording conditions, we started the recordings after an additional waiting period of at least $1 \mathrm{~h}$. Although we did not select neurons on the basis of their response properties (fast spiking/large amplitude, small spike-waveform width), there is a tendency in extracellular recordings using large and (relatively) low-impedance electrodes to record from large pyramidal neurons in layer 5 because of a recording sampling bias ${ }^{46}$. This tendency may be further strengthened by our spike sorting procedure, in which we only selected well-isolated single units with a high signal-to-noise ratio.

Behavioral procedure. The behavioral procedure and stimuli that we used were described previously ${ }^{25}$. Briefly, the monkeys performed a delayed matching-tosample task. They initiated a trial start by grasping a lever and fixating on a small fixation spot on the center of the screen. After successful fixation of 1,000 ms, a first stimulus appeared on the screen for $250 \mathrm{~ms}$ (sample stimulus), which was followed by a delay period of $1,500 \mathrm{~ms}$ during which the monkey held fixation. After the delay, a second stimulus (test stimulus) was presented for $600 \mathrm{~ms}$. The monkeys were rewarded for a lever release during this time window whenever the test matched the sample. Whenever they did not match, the monkeys' task was to withhold the lever release until, after an additional $200 \mathrm{~ms}$, a second test stimulus appeared that always matched the sample. This procedure ensured that the monkey had to initiate a behavioral response on every trial. The monkeys were rewarded with juice for every correct trial. In each experiment, $50 \%$ of the trials were match trials and $50 \%$ were non-match trials.

In each experiment, a set of three or four natural images was presented. The stimuli were $7^{\circ} \times 7^{\circ}$ in size and presented at the center of gaze on a monitor (Intergraph 21sd107) with linear luminance response (gamma corrected) at a distance of approximately $110 \mathrm{~cm}$. Prior to the recording sessions, the monkeys had been familiarized with the images and we ensured that monkeys did not show performance changes as a result of further learning ${ }^{47}$. The images were chosen from the Corel-Photo-CD Corel Professional Photos, comprising a collection of natural images showing birds, flowers, monkeys and butterflies in their natural surroundings and were degraded with visual noise to various degrees, including showing stimuli that only contained visual noise on approximately $10 \%$ of the trials, to ensure motivation of the monkeys.

To assess behavioral performance, we first calculated psychophysical performance (percent correct responses) at each degradation level for each monkey individually per recording session and subsequently averaged the performance across sessions ( $N=20$ and 11 for monkeys 1 and 2, respectively). We subsequently fitted a psychometric function (logistic function).
Data analysis. All of the data analyses were performed with custom software written in Matlab (MathWorks). All of the spectral analyses were based on the same time-frequency decomposition using complex Morlet wavelets. The LFP was resampled at $200 \mathrm{~Hz}$ and normalized by subtracting the mean waveform and dividing the result by the s.d. over trials, respectively. We extracted the instantaneous amplitude and analytical phase as a function of time and frequency by convolving the raw real-valued time series $x(t)$ with the complex Morlet wavelet $w\left(t, f_{0}\right)$ to obtain the complex output signal $y\left(t, f_{0}\right)$, also denoted as the analytic signal, where $f_{0}$ denotes the desired center frequency of the wavelet function. We used a value of $c=7$ wavelet oscillations as proposed previously 47,48 . The center frequencies $f_{0}$ were $2.63,3.03,3.48,4,4.6,5.27,6.06,6.96,8,9.18,10.55$, $12.12,13.92,16,18.37,21.11,24.25,27.85,32,36.75,42.22,48.5,55.7,64,73.51$, 84.44 and $97 \mathrm{~Hz}$.

To analyze the amplitude spectrum, we discarded stimulus-evoked components of the oscillations by subtracting the (evoked) mean waveform across trials from single trials before applying wavelet transform filters. We subsequently averaged the log-transformed spectra across individual sites. We obtained baseline and delay spectra by averaging the amplitude spectra in a $1,000-\mathrm{ms}$ window preceding the sample stimulus (baseline) and across the last 1,000 ms preceding the test stimulus, respectively. The latter time window was chosen to ensure that the frequency content was not contaminated by stimulus-evoked activity. For all analyses, unless otherwise stated, the same time windows were chosen for baseline and delay.

We quantified phase locking between individual pairs of LFP sites $(m, n)$ by computing the phase-locking value ${ }^{26}$ across the entire trial period. The PLV for two recording channels $\mathrm{m}$ and $\mathrm{n}$ at a particular center frequency $f_{0}$ and time $t$ is defined as

$$
P L V_{m n}\left(t, f_{0}\right)=\frac{1}{K}\left|\sum_{k=1}^{K} e^{i\left(\varphi_{k}^{m}\left(t, f_{0}\right)-\varphi_{k}^{n}\left(t, f_{0}\right)\right)}\right|
$$

where $K$ denotes the number of trials, and $\varphi_{k}^{m}\left(t, f_{0}\right)$ and $\varphi_{k}^{n}\left(t, f_{0}\right)$ denote the instantaneous phases of the $k$-th trial of the two channels that were computed using the wavelet transform with center frequency $f_{0}$. Thus, the PLV measures the degree of similarity of the phase difference $\Phi_{k}^{m n}\left(t, f_{0}\right)=\varphi_{k}^{m}\left(t, f_{0}\right)-\varphi_{k}^{n}\left(t, f_{0}\right)$ across different trials $k$ and ranges in the interval $0 \leq P L V_{m n} \leq 1$. The higher the similarity of phase, differences across trials, the higher is the PLV. As the phase-locking measure yields a single value, which is obtained across multiple trials, we tested for significance between baseline and delay in single electrode pairs using a bootstrapping procedure. We obtained confidence intervals for the baseline and delay by generating 1,001 bootstrap samples from the original trial set (with replacement), of the same size as the original data. A PLV during the delay was considered to be significantly different from baseline only if the average PLV over all bootstrap samples was above or below the $99.9^{\text {th }}$ percentile of baseline distribution of the PLV. To obtain the proportion of significantly phase-locked LFP pairs in each frequency band, we averaged the proportions of significant pairs that we obtained across frequencies in the band of interest, that is, $3-9 \mathrm{~Hz}$ for theta, $16-36 \mathrm{~Hz}$ for beta and $47-97 \mathrm{~Hz}$ for gamma. To compare phase locking between delay and baseline across the population of LFP pairs, we first subtracted the mean phase locking during baseline from every phase locking value across the entire trial period per pair, divided by the s.d. across pairs and subsequently averaged across all pairs. This procedure resulted in the normalized $z$ score-transformed phase-locking values.

To investigate spike-phase locking in each area, we excluded LFP-unit pairs recorded at the same electrode site and obtained 601 spike-LFP pairs in V4 (monkey 1, 426; monkey 2, 175), and 397 pairs in $\operatorname{lPF}$ (monkey 1, 216; monkey 2, 181). To examine inter-area locking, we paired 110 lPF LFP channels (monkey 1, 67; monkey 2,43 ) with the spiking activity of 167 simultaneously recorded V4 units (monkey 1, 114; monkey 2, 53) and 124 V4 LFP channels (monkey 1, 79; monkey 2,45 ) with the spiking activity of 130 simultaneously recorded IPF units (monkey 1,69; monkey 2, 61). This resulted in 660 lPF LFP-V4 unit pairs (monkey 1, 458; monkey 2, 202) and 593 V4 LFP-lPF unit pairs (monkey 1, 335; monkey 2, 258). We did not select channels or units on the basis of any criteria. Thus, our data represent a completely unbiased sample of LFP and SUA in both regions.

We tested for circular non-uniformity of the distribution of spikes with respect to the phase of the theta oscillation. Each spike was assigned its respective phase value of the simultaneous LFP oscillation (the phase was obtained using the same 
wavelet decomposition as described above). Because the window is usually no integer multiple of the oscillation length of $1 / f_{0}$, some phase values can occur more often than others, which results in non-uniformity per se. To account for that, we normalized the spike counts for each bin using the number of occurrences of each particular phase bin during the considered window for each trial. If spikes occur more often at particular phases, this procedure results in a non-uniform distribution of phase values across phase bins. We then applied the Rayleigh test for circular uniformity to test whether the spikes were significantly locked to the theta oscillation. We defined significant phase locking to be present for pairs for which we obtained a value of at least $\ln (z)=1.09$, which approximately corresponds to a value of $P=0.05$ for distributions containing at least 50 spikes $^{49}$.

To estimate concentration parameter $\kappa$ and mean direction $\mu$ during the baseline, we cut out spike trains in a 500-ms window preceding the sample stimulus (baseline) and a 500-ms window during the delay period that were centered on the maximal troughs of the filtered theta oscillations (frequencies ranging from 3 to $9 \mathrm{~Hz}$ ). We chose the central $500 \mathrm{~ms}$ of the delay period to not contaminate spiking activity by either the sensory response after sample stimulus onset or spiking activity related to test stimulus, thus keeping spike rates between baseline and delay as comparable as possible. In these windows, spikes were binned according to the phase of a sine wave of frequency $f_{0}$ mapped onto the extracted cycle. This procedure was repeated for each trial and the resulting windows were subsequently stacked up to form theta-triggered spike train rasters (as seen in Fig. 4a). Spikes were then summed across trials to obtain histograms containing the spike counts as a function of phase bin. We subsequently fitted von Mises density functions with mean direction $\mu$ and concentration parameter $\kappa$ to the spike distribution across phase bins.

We compared the median concentration parameter $\kappa$ between groups of neurons that could potentially vary with respect to mean spike rate. To account for these differences, we also compared $\kappa$ between groups of units whose mean firing rate was matched.

To create two sets of prefrontal and V4 neurons with (approximately) the same mean firing rate, we selected the most active cells from V4 and the same number of least active cells from IPF. The number of cells selected was chosen such that the mean firing rates in the two selections were as similar as possible. For monkey 1 , the mean rates did not differ significantly between $\mathrm{V} 4$ and $\mathrm{lPF}$ during delay (mean rate $\mathrm{V} 4,18.3 \mathrm{~Hz}$; mean rate $\mathrm{lPF}, 18.9 \mathrm{~Hz}$; independent sample $t$ test, $t=0.43$, $P>0.05)$. Thus, we did not correct for mean rates for units from this monkey. In contrast, for monkey 2 , the mean spike rate during delay was significantly larger for prefrontal than for V4 units (mean rate V4, 23.4 Hz; mean rate prefrontal cortex, $33.7 \mathrm{~Hz}$; independent sample $t$ test, $t=4.9, P<0.01$ ). After the selection, the mean rates for units from V4 and $1 \mathrm{PF}$ were 25.3 and $25.4 \mathrm{~Hz}$, respectively (independent sample $t$ test, $t=0.05, P>0.05$ ). The same procedure was applied when we compared the median $\kappa$ values for the baseline versus the delay period in the group of prefrontal and V4 units. Prefrontal unit activity tended to be higher during the delay than during baseline (mean rate delay, 20.8; mean rate baseline, 19.6), but was only significant in monkey 1 ( $t$ test; monkey $1, t=5.8, P<0.01$; monkey $2, t=1.6, P>0.05)$. In $\mathrm{V} 4$, mean spike rates tended to be lower during the delay than the baseline period (mean rate delay, $16.6 \mathrm{~Hz}$; mean rate baseline, $21.5 \mathrm{~Hz}$ ). The difference was significant in both monkeys ( $t$ test; monkey $1, t=4.0$; monkey $2, t=5.6 ; P<0.01$ ).

For all analyses involving SUA and LFP signals from the same area, we only analyzed signals recorded simultaneously from neighboring electrodes, that is, at least $0.5 \mathrm{~mm}$ away. Thus, we avoided artifacts resulting from spillover of signals from SUA into the LFP signal recorded at the same electrode. This also guaranteed comparable conditions for interactions in and between the areas.

We assessed whether LFP-phase locking (and spike-phase locking) significantly differed between correct and incorrect trials by computing the $\mathrm{d}^{\prime}$ values between correct and incorrect sets of trials per pair. We only chose trials for which stimuli were shown at a visual noise level ( $45 \%$ coherence), as the monkeys made enough errors in this stimulus condition to reliably estimate phase locking from both correct and incorrect trials. Correct trials were defined as trials in which the monkeys correctly identified the previously shown stimulus (hit), and incorrect trials were defined as trials in which the animals did not (miss). To avoid confounding phase locking measurements with differences in trial numbers, we computed phase locking only across the minimum number of trials in each condition.

To estimate how variable PLVs would be for correct versus incorrect sets of trials, we first computed phase locking for different subsamples of trials for both conditions (199 repetitions) and then subtracted the mean across subsampled PLVs from correct trials from the mean across PLVs from incorrect trials. Subsequently, we divided the difference by the s.d. across all PLVs. d' values were computed for original datasets and datasets in which the trials were randomly exchanged between the correct and incorrect condition (shuffled). The $P$ values represent the average proportion of cases in which the shuffled $\mathrm{d}^{\prime}$ averaged across pairs during delay was higher than the original d' (Fig. 5d).

To correlate the difference in locking between correct and incorrect responses with session-to-session variations in performance, we assigned each V4-LFP pair its respective proportion of correct performance value of the session in which the pair was recorded and computed Spearman's rank correlation coefficient across all sessions/pairs from bootstrapped samples drawn with replacement from the original dataset. We report the mean and $95^{\text {th }}$ percentile confidence intervals of correlations across the bootstrapped samples $(N=999)$. To illustrate the dependency of $\mathrm{d}^{\prime}$ and performance further, we binned $\mathrm{d}^{\prime}$ data points using six equally spaced bins for the proportion of correct responses and averaged across d' values per bin.

46. Towe, A.L. \& Harding, G.W. Extracellular microelectrode sampling bias. Exp. Neurol. 29, 366-381 (1970).

47. Graimann, B. \& Pfurtscheller, G. Quantification and visualization of event-related changes in oscillatory brain activity in the time-frequency domain. Prog. Brain Res. 159, 79-97 (2006).

48. Tallon-Baudry, C. \& Bertrand, O. Oscillatory gamma activity in humans and its role in object representation. Trends Cogn. Sci. 3, 151-162 (1999).

49. Siapas, A.G., Lubenov, E.V. \& Wilson, M.A. Prefrontal phase locking to hippocampal theta oscillations. Neuron 46, 141-151 (2005). 